\title{
Suicídio entre Médicos e Estudantes de Medicina: Revisão de Literatura
}

\section{A Review of Literature on Suicide among Doctors and Medical Students}

\author{
Nathália Della Santa \\ Amaury Cantilino
}

\section{PALAVRAS-CHAVE}

- Saúde Mental;

- Educação Médica;

- Tentativa de Suicídio.

\section{KEYWORDS}

- Mental Health;

- Education, Medical;

- Suicide, Attempted.

Recebido em: 02/02/2015

Aprovado em: 16/02/2016

\section{ABSTRACT}

Objectives: This study aims to construct an integrative review of suicide among physicians and medical students based on an analysis of scientific articles on the subject. Methods: A search was conducted in the PubMed, Lilacs and SciELO databases using the keywords "suicide", "physicians", "doctors", and "students" between 2000 and 2014 in English, German, Portuguese, and Spanish. Results: The results indicated that suicide rates in this population are higher than those of the general population and other academic groups. The main causes identified in the study were a higher incidence of psychiatric disorders such as depression and substance abuse, and psychological distress related to specific situations involved with the profession, such as high workload, sleep deprivation, difficulty with patients, unhealthy environments, financial worries, and information overload. Conclusions: Suicide prevention is possible, but the problem is not receiving the necessary attention. Additional research on the subject is required to formulate policies and interventions for prevention and specific treatment for this population. 


\section{INTRODUÇÃO}

O suicídio é considerado um problema de saúde pública pela Organização Mundial da Saúde (OMS) ${ }^{1}$. Ele está entre as dez principais causas de morte na maioria dos países e na segunda ou terceira posição na população entre 15 e 34 anos de ida$\mathrm{de}^{2,3,1}$. Ainda de acordo com a OMS, nos últimos 45 anos, a taxa de mortalidade por suicídio aumentou $60 \%{ }^{2,3}$. Nesse mesmo período, os maiores coeficientes de suicídio mudaram da faixa mais idosa para as faixas mais jovens ${ }^{3,1}$. No Brasil, nos últimos 25 anos, a taxa de mortalidade por suicídio em jovens e adolescentes aumentou cerca de 30\%4. Em 2005, o Brasil registrou cerca de 8.550 mortes por suicídio. Esse número coloca o Brasil entre os dez países com as mais altas taxas de suicídio no mundo $\mathrm{o}^{2,3,5}$.

Os comportamentos suicidas podem ser classificados em três categorias: ideação suicida, tentativa de suicídio e suicídio consumado ${ }^{6}$. Ideias, desejos, declarações sobre querer morrer, planejamento da morte e o pensamento sobre como tal atitude iria influenciar as pessoas também fazem parte do espectro de comportamento suicida. Frequentes ou pouco frequentes, essas ações normalmente procuram resolver algo insuportável para o indivíduo e aparecem em escala de gravidade. Este gradiente vai desde a concepção até a consumação do suicídio ${ }^{6,7}$.

Frente a tal contexto sobre o tema, tem se destacado o aumento na taxa de suicídio em médicos, conhecida por ser mais elevada do que a da população em geral $^{8,9,10}$. Tal condição é alarmante, também, porque um estudo americano publicado em 2013 revelou que poucas pesquisas avaliaram as informações sobre comorbidades de saúde mental e estressores psicossociais que podem contribuir para o suicídio nessa classe profissional $^{9}$. Devido à importância desse tema ${ }^{11,9,10}$, o presente estudo tem como objetivo realizar uma revisão da literatura sobre suicídio entre médicos e estudantes de Medicina.

\section{MÉTODO}

Realizou-se uma busca bibliográfica para aferir os artigos sobre o tema proposto. Os artigos foram consultados nas bases de dados PubMed, SciELO e Lilacs. A pesquisa foi feita por meio do cruzamento entre os seguintes descritores: "suicide", "physicians", "doctors" e "students". Foram utilizados artigos escritos em inglês, português, espanhol e alemão, publicados nos últimos 180 meses, ou seja, de 2000 a 2014. Também foram usados livros que contemplam o tema e que puderam contribuir com a construção desta revisão, cartilhas de prevenção ao suicídio, assim como artigos que foram sugeridos, como associação ao tema, pela base de dados durante a captação dos artigos. Assim, foram incluídos artigos originais, pesquisas quantitativas e qualitativas, estudos retrospectivos, artigos de revisão sobre o tema e estudos de casos.

A pesquisa foi realizada em duas fases: (a) triagem de títulos e resumos: nesta fase, foram excluídos os artigos que não se adequavam à temática estudada; (b) após a primeira triagem dos títulos e resumos, foi verificada a existência de duplicidade dos artigos nas seleções das bases de dados, ou seja, se dois artigos iguais foram selecionados em bases de dados diferentes. Após essas duas triagens, os artigos selecionados foram lidos integralmente para a construção deste trabalho.

\section{RESULTADOS}

Com base na pesquisa bibliográfica, foram selecionados 17 artigos para a construção desta revisão integrativa (Quadro 1 e Tabela 1). Na base de dados do PubMed/Medline foram captados 59 artigos. Na primeira fase da pesquisa, 45 artigos foram excluídos por não se adequarem ao tema deste estudo. Destas pesquisas excluídas, os principais temas encontrados foram suicídio em jovens e adolescentes (nove artigos) e eutanásia/suicídio assistido (11 artigos). Quatorze artigos foram utilizados nesta pesquisa. No Lilacs foram captados nove artigos. Apenas um artigo foi utilizado na pesquisa. Um foi excluído por duplicidade e sete foram excluídos por não contemplarem o tema proposto.

Na base de dados SciELO foram encontrados 11 artigos no cruzamento dos descritores "suicide" e "doctors". Destes, apenas dois foram utilizados, e seus temas se referiam às possíveis causas e inquietações que estão fortemente relacionadas

\begin{tabular}{|c|c|c|c|c|c|}
\hline \multicolumn{6}{|c|}{$\begin{array}{l}\text { TABELA } 1 \\
\text { Etapas de seleção dos artigos para a revisão integrativa de literatura }\end{array}$} \\
\hline & Total de artigos captados & $\begin{array}{l}\text { 1a etapa: exclusão } \\
\text { por título e resumo }\end{array}$ & $\begin{array}{l}2^{\text {a }} \text { etapa: exclusão } \\
\text { por duplicidade }\end{array}$ & $\begin{array}{c}3^{3} \text { etapa: } \\
\text { leitura na íntegra }\end{array}$ & $\begin{array}{c}\text { Artigos } \\
\text { selecionados }\end{array}$ \\
\hline Pubmed & 59 & 45 & 0 & 14 & \multirow{3}{*}{$\begin{array}{l}14 \text { artigos originais } \\
3 \text { artigos de revisão }\end{array}$} \\
\hline Lilacs & 9 & 7 & 1 & 1 & \\
\hline SciELO & 11 & 9 & 0 & 2 & \\
\hline
\end{tabular}

\begin{tabular}{l|l}
\hline REVISTA BRASILEIRA DE EDUCAÇÃO MÉDICA \\
\hline 70 (4): 772 - $780 ; 2016$
\end{tabular}




\begin{tabular}{|c|c|c|c|c|}
\hline \multicolumn{5}{|c|}{$\begin{array}{l}\text { QUADRO } 1 \\
\text { Artigos captados para a revisão integrativa de literatura }\end{array}$} \\
\hline Título do trabalho & Autores & Ano & País & Desenho \\
\hline Suicide in medical students: case series & Kamski L et al. & 2012 & Alemanha & Artigo original \\
\hline $\begin{array}{l}\text { Risk factors at medical school for later severe depression: a 15- } \\
\text { year longitudinal, nationwide study (Nordoc) }\end{array}$ & Støen Grotmol K et al. & 2013 & Noruega & $\begin{array}{l}\text { Artigo original - } \\
\text { pesquisa longitudinal }\end{array}$ \\
\hline $\begin{array}{l}\text { Depressive symptoms in medical students and residents: } a \\
\text { multischool study }\end{array}$ & Goebert D et al. & 2009 & Estado Unidos & $\begin{array}{l}\text { Artigo original - } \\
\text { pesquisa transversal }\end{array}$ \\
\hline $\begin{array}{l}\text { Psychosocial health risk factors and resources of medical } \\
\text { students and physicians: a cross-sectional study }\end{array}$ & Voltmer et al. & 2008 & Alemanha & $\begin{array}{l}\text { Artigo original - } \\
\text { pesquisa transversal }\end{array}$ \\
\hline $\begin{array}{l}\text { Application of an interpersonal-psychological model of suicidal } \\
\text { behavior to physicians and medical trainees }\end{array}$ & Cornette MM, et al. & 2009 & & Artigo de revisão \\
\hline $\begin{array}{l}\text { Not enough help for themselves - the risk of physicians to } \\
\text { commit suicide }\end{array}$ & Püschel K, Schalinski S. & 2006 & Alemanha & Revisão de literatura \\
\hline Suicidal tendencies of physicians - an overview & Reimer C et al. & 2005 & Alemanha & $\begin{array}{l}\text { Revisão sistemática } \\
\text { de literatura }\end{array}$ \\
\hline $\begin{array}{l}\text { The process of suicidal planning among medical doctors: } \\
\text { predictors in a longitudinal Norwegian sample }\end{array}$ & Tyssen R et al. & 2004 & Noruega & Artigo original \\
\hline $\begin{array}{l}\text { Suicidal ideation and medical profession: from medical students } \\
\text { to hospital physicians }\end{array}$ & Martinac M et al. & 2003 & Bosnia & Artigo original \\
\hline $\begin{array}{l}\text { Suicidal ideation among medical students and young } \\
\text { physicians: a nationwide and prospective study of prevalence } \\
\text { and predictors }\end{array}$ & Tyssen R et al. & 2001 & Noruega & Artigo original \\
\hline $\begin{array}{l}\text { The impact of job stress and working conditions on mental } \\
\text { health problems among junior house officers. A nationwide } \\
\text { Norwegian prospective cohort study }\end{array}$ & Tyssen R et al. & 2000 & Noruega & Artigo original \\
\hline $\begin{array}{l}\text { Details on suicide among US physicians: data from the National } \\
\text { Violent Death Reporting System }\end{array}$ & Gold KJ et al. & 2013 & Estados Unidos & $\begin{array}{l}\text { Artigo original } \\
\text { retrospectivo }\end{array}$ \\
\hline $\begin{array}{l}\text { Suicidal ideation among students enrolled in healthcare training } \\
\text { programs: a cross-sectional study }\end{array}$ & Alexandrino-Silva $C$ et al. & 2009 & Brasil & Artigo original \\
\hline Suicide in doctors: a psychological autopsy study & Hawton et al. & 2004 & Inglaterra & $\begin{array}{l}\text { Artigo original } \\
\text { retrospectivo }\end{array}$ \\
\hline $\begin{array}{l}\text { Prevalência de sintomas depressivos em estudantes de } \\
\text { Medicina }\end{array}$ & Vallilo et al. & 2011 & Brasil & Artigo original \\
\hline Traços de ansiedade entre estudantes de Medicina & Baldassin et al. & 2006 & Brasil & Artigo original \\
\hline A angústia na formação do estudante de Medicina & Quintana et al. & 2007 & Brasil & Artigo original \\
\hline
\end{tabular}

ao comportamento suicida em profissionais de saúde e prevenção de suicídio em médicos.

Entre os artigos selecionados para leitura completa, as principais temáticas encontradas foram as taxas superiores de suicídio dos estudantes de Medicina em comparação às de outras áreas de formação, fatores de risco apresentados para o comportamento suicida nesta população e na população médica, e prevenção do comportamento suicida. Assim, a exposição dos dados foi organizada nas categorias a seguir (Quadro 1).

\section{Suicídio e ideações suicidas em estudantes de Medicina e} médicos

Revisões sistemáticas e estudos longitudinais de literatura feitos sobre o suicídio, especificamente em médicos, identificam que as taxas de suicídio nessa população são mais elevadas do que as da população geral e de outros grupos acadêmicos ${ }^{9-12}$. Um estudo realizado na Alemanha em 2012 menciona que a taxa de suicídio em médicos é de três a cinco vezes maior do que a da população geral ${ }^{11}$. 
Kamski et al. ${ }^{11}$, em pesquisa realizada em uma universidade austríaca, identificaram 14 suicídios em estudantes de Medicina de janeiro de 2006 a agosto de 2011. Esses números refletem uma média de três suicídios por ano ${ }^{11}$. Entre os 14 casos, três foram mulheres e 11 homens. Com relação aos métodos utilizados, seis casos foram por enforcamento/asfixia, cinco casos por salto de uma grande altura, dois por intoxicação e um por salto em frente a um trem. Em 50\% desses casos, os estudantes já haviam tido acompanhamento psiquiátrico e dois estavam em tratamento na época da morte ${ }^{11}$.

Já com relação à ideação suicida, os dados são mais difíceis de pesquisar e registrar, já que nem sempre são expostos pelos indivíduos que pensam sobre isso e nem identificados pelas pessoas com quem convivem ${ }^{13}$. Um estudo em 2009 nos Estados Unidos com 2 mil estudantes de Medicina e residentes indicou que quase $6 \%$ dessa amostra relataram ideação suicida. Essa pesquisa revelou também que as maiores taxas de ideação suicida foram encontradas nos estudantes que apresentaram depressão maior ${ }^{13}$.

Dalgalarrondo et al. ${ }^{14}$ destacam a correlação entre ideação suicida e efetivação do suicídio. Estima-se que $60 \%$ dos indivíduos que se suicidam apresentavam previamente ideações ${ }^{14}$. Refere-se que a gravidade e a intensidade dos pensamentos suicidas estão diretamente correlacionadas com as tentativas de suicídio, que representam, por sua vez, o maior fator de risco para o suicídio completo ${ }^{14}$.

Como exemplo dessa relação entre ideação suicida e suicídio efetivo, destaca-se um estudo na Universidade de Mostar, na Bósnia. Na avaliação da ideação suicida de 200 alunos, esse estudo identificou que 9,16\% dessa amostra completaram o comportamento suicida, levando os estudantes à morte ${ }^{12}$.

Nesse contexto, chama-se a atenção para a pesquisa de Tyssen et al. ${ }^{15}$, em um estudo prospectivo com 5.522 estudantes na Noruega, revelando que $14 \%$ da amostra apresentaram pensamentos suicidas. Deste universo, $8 \%$ apresentaram planejamento para o suicídio e 1,4\% tentou o suicídio. Os preditores identificados nesta população foram falta de controle sobre as situações, traços de personalidade, estado civil solteiro, eventos negativos na vida e sofrimento mental, especialmente depressão e ansiedade ${ }^{15}$.

\section{Fatores que influenciam os comportamentos suicidas}

Kamski et al. ${ }^{11}$ registraram que houve dificuldade para colher informações a respeito do assunto em sua pesquisa. Isto pode ser explicado pelo tabu relacionado ao tema. Pontua-se que não foi reconhecido apenas um fator específico que justifique tais taxas, mas possivelmente uma junção de aspectos pessoais e ambientais ${ }^{11,13}$.
Com relação a essa junção de fatores que podem influenciar a ideação e o comportamento suicida, a maior parte dos artigos indica transtornos psiquiátricos, como depressão e abuso de substâncias, e fatores externos, como questões relacionadas às condições de trabalho, pressão psicológica, alta carga de trabalho, etc. ${ }^{13}$.

Uma pesquisa da Universidade de Oxford publicada em $2004^{10}$ investigou fatores relacionados ao suicídio em médicos. A pesquisa foi realizada por meio de autópsia psicológica de 38 médicos que cometeram suicídio. Dentre esses, 25 apresentavam algum tipo de transtorno mental, principalmente depressão e abuso de álcool. Os problemas enfrentados por esses médicos foram identificados como relacionados ao trabalho (25), aos relacionamentos interpessoais (14), financeiros (10) e a um conjunto desses fatores $(12)^{10}$.

Em convergência com a pesquisa mencionada, uma revisão de literatura realizada em $2005^{16}$ analisou os artigos originais sobre o tema com o objetivo de fornecer uma visão geral sobre as taxas de suicídio e tendências suicidas em médicos. Essa pesquisa identificou que uma das possíveis causas do aumento da taxa de suicídio nessa população é o aumento da prevalência de transtornos depressivos, bem como o abuso de substâncias ${ }^{16}$.

Neste sentido, além da superioridade nas taxas de suicídio, estudos também indicam que há evidências de que os estudantes de Medicina e residentes têm mais possibilidade de sofrer de depressão do que alunos de outras pós-graduações e adultos jovens em geral ${ }^{13,17}$. A escola médica é reconhecida por inúmeras pesquisas como desencadeadora de estresse e como influência negativa no bem-estar físico e psicológico, e no desempenho acadêmico dos estudantes de Medicina. Os transtornos mentais, segundo a literatura, podem estar presentes em até $50 \%$ dessa população ${ }^{13,16}$.

Dentre os transtornos mentais, a depressão é uma doença que tem sido estudada há muito tempo no meio médico. Um estudo $^{17}$ realizado com 400 estudantes de Medicina na cidade de Uberlândia, no Brasil, identificou que 79\% dos alunos apresentaram algum sintoma depressivo, sendo $29 \%$ com grau leve, 31\% moderado e 19,25\% grave. A patologia é frequentemente associada ao fenômeno do suicídio. Os principais fatores de risco para esse transtorno encontrados nesta população são grande carga de trabalho, privação do sono, dificuldade com pacientes, ambientes insalubres, preocupações financeiras e sobrecarga de informações ${ }^{13}$. Esses estressores podem influenciar negativamente o desempenho acadêmico, a saúde física e o bem-estar psicológico dos estudantes, tornando-os mais vulneráveis emocionalmente ${ }^{13}$.

Sabe-se que cerca de $90 \%$ das pessoas que cometem suicídio são diagnosticadas com transtornos mentais ${ }^{18}$. $\mathrm{O}$ desen- 
volvimento de depressão e sintomas de ansiedade está relacionado com o surgimento e a manutenção de ideações suicidas ${ }^{18}$. Apesar de haver muitos estudos sobre os transtornos mentais e médicos e o estresse na formação médica, a associação entre essas condições e o suicídio não tem sido amplamente estudada nessa população específica ${ }^{9,18}$.

Na tentativa de elucidar essa relação entre os transtornos psiquiátricos e suicídio em médicos, destaca-se o estudo de Alexandrino-Silva et al. ${ }^{18}$. Essa pesquisa investigou a existência de pensamentos suicidas, sintomas depressivos e sintomas de desesperança entre estudantes de Medicina, Farmácia e Enfermagem. O resultado dessa pesquisa revelou que os pensamentos de desesperança foram mais frequentes em estudantes de Medicina do que nos outros dois cursos. Também foi identificada a existência de correlação positiva entre o risco de suicídio e a presença de sintomas depressivos e de desesperança ${ }^{18}$.

Os transtornos psiquiátricos do espectro da ansiedade também são frequentes nesta população. Uma pesquisa realizada com 603 estudantes de Medicina de diversos períodos no Brasi $^{19}$ revelou que 20,1\% da amostra apresentaram alto grau de ansiedade e 79,9\% sugeriram ansiedade média. Nenhum estudante apresentou escores de baixa ansiedade. $\mathrm{O}$ maior percentual de traço de ansiedade alta foi encontrado no sexto ano $(26,8 \%)$ e o menor no terceiro ano $(11,6 \%)$. O estudo sugere que o curso médico está intensamente associado ao desenvolvimento de quadros ansiosos nos estudantes ${ }^{19}$.

Uma pesquisa realizada por Tyssen et al. ${ }^{20}$ revelou que a vivência médica e os aspectos peculiares da profissão não influenciam mais do que as outras formações em saúde. O estudo revelou que, numa população de 631 médicos na pós-graduação, os preditores aparentes na escola médica eram semelhantes aos fatores de risco de suicídio da população geral. Preditores como sintomas graves de depressão, traços de personalidade e estresse na vida pessoal foram encontrados, ao passo que fatores contextuais relacionados ao curso de Medicina não foram.

Em contrapartida, Meleiro ${ }^{21}$ refere e destaca em revisão bibliográfica fatores externos que podem favorecer a vulnerabilidade psicológica dos médicos e estudantes de Medicina em especial. Além da alta incidência de morbidades psiquiátricas e do uso de drogas já referidas nesta população, mencionam-se fatores externos, como, por exemplo, dependência financeira em idade avançada, pressão por alto rendimento nos estudos, intensa atividade intelectual, problemas de identidade e medos ${ }^{11}$. Parece ser crucial para a decisão de se suicidar a combinação entre as circunstâncias da vida, a saúde mental do indivíduo e eventos estressantes ${ }^{1}$. Além disso, pontua-se como aspecto facilitador do fenômeno nesta população a disponibilidade e a oportunidade de acesso a meios de cometer o ato suicida e o conhecimento científico desenvolvido ${ }^{1}$.

A respeito das facilidades de acesso mencionadas, um estudo realizado com estudantes de Berlim em $2010^{16}$ demonstrou que cerca de um terço dos 850 alunos participantes da pesquisa usa algum tipo medicação de forma regular como uma fuga dos problemas através de fármacos ${ }^{16}$.

Além do conhecimento sobre as formas e dos fatores que influenciam a decisão, o processo pelo qual o indivíduo passa também é um aspecto que deve ser investigado. Tyssen et al. ${ }^{20}$ pesquisaram sobre o processo que leva o estudante de pós-graduação de Medicina ao suicídio. A pesquisa refere que poucos estudos têm se debruçado sobre este processo, que se traduz em um processo continuum de gravidade ${ }^{20}$. Esse processo é iniciado com pensamentos de que a vida não vale a pena, ideações suicidas de forma geral, seguidas pelo ato de considerar seriamente o suicídio como uma real possibilidade, planejar e, finalmente, tentar o suicídio. Os autores destacam especialmente o planejamento suicida, uma vez que, na população geral, cerca de $72 \%$ evoluem do planejamento para a tentativa real de suicídio ${ }^{20}$.

Ainda a despeito dos fatores externos peculiares à população em estudo, evidenciam-se as feridas narcísicas profundas, o medo de decepcionar e ter a reputação abalada socialmente frente à criação de expectativas irreais a respeito do desempenho pessoal e necessidade de apresentar alto rendimento ${ }^{11}$. Na Alemanha, em 2006, uma pesquisa qualitativa, baseada em estudos e meta-análises, evidenciou que as ideações e comportamentos suicidas comuns em médicos são fundamentados nas dificuldades que eles têm de corresponder à representação social dos médicos ${ }^{22}$. Essa representação é simbolizada pelo comportamento de equilíbrio, de ajuda e de disponibilidade. Essa expectativa pode corroborar a dificuldade que esses profissionais têm na identificação de seus próprios problemas emocionais e, consequentemente, na solicitação de ajuda aos colegas e nas intervenções médicas necessárias ao seu tratamento ${ }^{22}$. Esse movimento pode ser compreendido como receio da discriminação laboral e pessoal do profissional; pensar, planejar e cometer o suicídio vão de encontro à imagem e à principal função do médico que é defender a vida sob qualquer circunstância ${ }^{22}$

Para além desses dados e resultados, Püschel e Schalinski ${ }^{22}$ chamam atenção para o movimento de subnotificação dos suicídios no meio médico, alterando os certificados de óbito em função da proteção contra a imagem negativa que o ato suicida pode causar, condição esta que prejudica a notificação e informação quanto à gravidade do problema na classe médica. 


\section{Prevenção do suicídio}

Frente a tal situação do fenômeno do suicídio, o Conselho Federal de Psicologia do Brasil ${ }^{23}$ publicou em 2013 uma cartilha sobre o assunto. Embora não seja específica para o meio médico, a cartilha objetiva servir como um norteador de ações futuras no campo da psicologia sobre o tema e auxiliar na idealização de políticas públicas que busquem prevenir e assistir à questão. Esse trabalho foi constituído com base numa perspectiva social-psicológica sobre o fenômeno ${ }^{23}$.

Além dessa cartilha, o Conselho Federal de Medicina, junto com a Associação Brasileira de Psiquiatria, elaborou a cartilha intitulada "Suicídio: informando para prevenir"24. Ela foi construída com base no pressuposto de que o suicídio pode ser prevenido desde que os profissionais de saúde de todos os níveis de atenção estejam preparados para identificar e reconhecer fatores de risco ${ }^{24}$. Essa cartilha orienta médicos e profissionais da área de saúde em geral sobre a condução frente às situações de risco e auxilia na identificação de indícios de possíveis comportamentos suicidas nos pacientes ${ }^{24}$.

Nesses termos, Meleiro ${ }^{21}$ também ratificou que os suicídios podem ser reconhecidos e prevenidos em médicos. Analisando uma população de suicidas, foi possível verificar que, meses antes da morte, colegas próximos identificaram mudanças comportamentais, aumento da indecisão, desorganização e quadros depressivos, assim como identificado na população geral $^{21}$.

Na cartilha formulada pelo Conselho Federal de Psicologia, a prevenção é vinculada diretamente ao fenômeno e é considerada algo possível de realizar. A proposta parte do pressuposto de que a ideia de retirar a própria vida pode ser comunicada por meios verbais e não verbais, desde o ato de planejar até a tentativa do suicídio ${ }^{23} \mathrm{e}$, portanto, é passível de intervenções por profissionais preparados.

Mantendo-se em mente que a ideação suicida é o principal fator de risco para o suicídio completo, a identificação desses pensamentos e mudanças comportamentais nesta população (médicos e estudantes de Medicina) é fundamental para que se possa intervir e construir políticas específicas de prevenção ao suicídio para essa população.

De forma geral, o fenômeno do suicídio é um problema de saúde pública e de grande relevância nesse contexto. Segundo o Conselho Federal de Psicologia, embora os aspectos que contribuam para o suicídio variem entre grupos e populações específicos, os principais grupos de risco são os jovens, idosos e grupos socialmente isolados ${ }^{23}$. Ou seja, sugere-se que o isolamento social pode interferir na ideia, no planejamento e na execução do suicídio. Apesar de anualmente cerca de um milhão de pessoas cometer suicídio no mundo e de o suicídio estar entre as dez principais causas de morte em todo o mundo, questiona-se o pequeno número de estudos relacionados ao tema na população médica. A propósito, essa população também está suscetível ao isolamento social causado por longos anos de formação, grande volume de estudo e exaustiva carga acadêmica e de trabalho. Destaca-se o pequeno número de pesquisas feitas na área. Na presente pesquisa, do universo de 79 artigos selecionados na primeira fase da pesquisa, foram selecionados apenas 17 que discorreram especificamente sobre esse tema.

\section{DISCUSSÃO}

Nota-se que ainda hoje o tema do suicídio é um tabu na sociedade. Desde o início do curso médico, os estudantes aprendem a ver a morte como sua maior adversária, que ela deve ser combatida e, se possível, vencida em nome da ciência e da competência ${ }^{25}$. Essa lógica aprendida parece ser a vigente no meio médico e pode dificultar o debate e a exposição de profissionais que se posicionam contra ${ }^{22,25}$. Mesmo com dados alarmantes e superiores aos da população geral, foram ainda encontrados nos artigos analisados registros de subnotificações das mortes por suicídio na área médica. Essas subnotificações podem ser compreendidas como uma forma de proteção e de evitar a exposição do colega suicida ${ }^{22}$.

Nos artigos selecionados, foi consenso que a decisão de se suicidar resulta de um conjunto de fatores externos, orgânicos e circunstanciais. Embora o número de estudos específicos sobre o tema seja pequeno, em praticamente todos eles os preditores encontrados foram semelhantes: existência de transtornos psiquiátricos, como depressão, ansiedade e abuso de substâncias, e questões externas referentes aos hábitos e vivências peculiares da população médica. Apesar de essas causas estarem claras no estudo da literatura, foram encontrados poucos estudos que relatam projetos ou programas de apoio a essa população com modelos de intervenção.

Os poucos estudos encontrados se detiveram em analisar as taxas de suicídio e a existência de ideação suicida nos estudantes de Medicina e médicos formados, mas há uma defasagem na forma de intervenção nessa população frente a essas constatações. Os artigos destacam a importância da identificação dos diagnósticos e tratamento dos transtornos mentais. Essa intervenção é de extrema importância, mas é a conduta indicada para todos, já que cerca de $90 \%$ das pessoas que cometem suicídio são diagnosticadas com transtornos mentais ${ }^{18}$. Questionam-se, então, as intervenções que poderiam ser feitas de forma específica para a população médica, uma vez que ela apresenta taxas de suicídio e ideação suicida superiores às da população geral ${ }^{8,9,16}$. 
Em busca de compreender esse fenômeno e identificar intervenções possíveis e eficazes para o problema, reflete-se sobre o processo de formação médica. Esse processo implica, de forma significativa, a falta de tempo para o lazer e para contato com os amigos, família e parceiros(as) amorosos(as), grande número de perdas durante o curso, desconstrução da idealização da onipotência médica, crescente consciência dos problemas existentes na profissão, além de convivência com o clima de competição por melhores notas e vagas ${ }^{26}$.

Todos esses fatores podem influenciar negativamente a saúde mental do educando. Esse cenário, além de contribuir para o desenvolvimento de transtornos psicológicos ${ }^{26}$, que, segundo a literatura, podem estar presentes em até 50\% dessa população ${ }^{19}$, não colabora com a construção e manutenção de boas relações sociais e afetivas, que podem estar diretamente relacionadas à possibilidade de desinteresse pela vida.

O processo de formação médica se inicia com a seleção para o ingresso nas faculdades de Medicina. Esta seleção ocorre por meio de notas que avaliam a capacidade intelectual e o aprendizado teórico dos estudantes. Porém, existem outros aspectos não menos importantes que não são avaliados nesse processo, mas que influenciam diretamente o sucesso acadêmico dos estudantes e o sucesso profissional dos médicos. Habilidades sociais, como empatia, sociabilidade e tolerância à frustração, são exemplos dessas características.

A formação acadêmica parece ainda estar voltada para o desempenho intelectual. Apesar de disciplinas como Psicologia Médica já estarem presentes na maioria dos currículos médicos, parece haver ainda certa negligência com a inteligência emocional e as habilidades sociais necessárias ao crescimento profissional e ao bem-estar psicológico do indivíduo no decorrer do seu ofício ${ }^{11}$. Nesse sentido, o treinamento de habilidades sociais surge como uma possibilidade de intervenção para atuar nesse contexto, a fim de colaborar com a formação humana, social e emocional dos profissionais.

\section{CONCLUSÃO}

O presente estudou buscou fazer uma revisão integrativa sobre o suicídio em médicos e estudantes de Medicina. De forma geral, as pesquisas indicam que as taxas de suicídio nessa população são maiores do que as da população geral e de outros grupos acadêmicos, e enumeram fatores que justificam esses dados.

Esse aumento pode ser explicado pela maior incidência de transtornos psiquiátricos, como depressão, transtornos de ansiedade, abuso de álcool e substâncias, por exemplo. O fácil acesso e o maior conhecimento sobre o funcionamento fisiológico humano podem facilitar a efetivação do comportamento suicida. Além disso, a imagem social de equilíbrio e de apoio dificulta a solicitação de ajuda pelos médicos, impedindo a identificação dos transtornos e o auxílio com relação às insatisfações com a própria vida e às ideações suicidas nessa população.

A prevenção do suicídio é possível. Os estudos sobre a ideação suicida em médicos e estudantes de Medicina estão elucidando as causas, influências sociais, processos cognitivos e de tomada de decisão de finalizar a própria vida. As formas de intervenção consistem no diagnóstico e tratamento dos transtornos psicológicos comumente presentes na população e uma mudança no processo de formação médica que valorize e destaque as habilidades sociais e emocionais a fim de contribuir com o bem-estar do profissional no seu ofício.

De forma geral, o estudo pretendeu fornecer dados, mesmo que bibliográficos, para maior compreensão do fenômeno e, consequentemente, auxiliar na elaboração de intervenções clínicas e terapêuticas, assim como na construção de programas e políticas educativas de prevenção do suicídio na área profissional da saúde em geral.

Novas pesquisas e projetos são necessários para contribuir com mais informações e melhor compreensão dos processos psicológicos envolvidos no fenômeno. Seria interessante estudar profundamente as habilidades sociais nessa população, principalmente a empatia. Além disso, estudos que possam identificar as principais causas e estímulos psicológicos ao suicídio nos médicos também seriam importantes para a construção de intervenções mais eficazes. Por fim, sugere-se também a aplicação de programas de apoio que possam avaliar, de forma prática, a eficácia de intervenções específicas para o problema em tela.

\section{REFERÊNCIAS}

1. World Health Organization. The World Health Report 2003: shaping the future. Geneva: World Health Organization; 2003

2. Gonçalves LRC, Goncalves E, Oliveira Junior LB. Determinantes espaciais e socioeconômicos do suicídio no Brasil: uma abordagem regional. Nova econ.[online]. 2011;21(2):281-316.

3. Botega NJ, Marín-León L, Oliveira HB, Barros MBA, Silva VF, Dalgalarrondo P. Prevalências de ideação, plano e tentativa de suicídio: um inquérito de base populacional em Campinas , São Paulo. 2009;25(12):2632-8.

4. Bertolote JM. O Suicídio e sua prevenção. São Paulo: ed. Unesp; 2012.

5. Instituto Brasileiro de Geografia e Estatística. Tábuas completas de mortalidade - 2006. Disponível em: http:/ / www. 
ibge.gov.br/home/presidencia/noticias/noticia_impressao.php?id_noticia=1043.

6. Keller M, Guevara S. Flexibilidade na resolução de problemas em tentadores de suicídio. J Bras Psiquiatr. 2005;54(2):128-36. Disponível em: http://www.bvsde. paho.org/bvsacd/cd52/keller.pdf.

7. Ores LC, Quevedo LA, Jansen K, Carvalho AB, Cardoso TA, Souza LDM, et al. Risco de suicídio e comportamentos de risco à saúde em jovens de 18 a 24 anos : um estudo descritivo. 2012;28(2):305-12.

8. Katherine J, Gold MD, Ananda SEN, Thomas L, Schwenk M. Details on suicide among U.S. physicians: Data from the National Violent Death Reporting System. Gen Hosp Psychiatry. 2013;35(1):45-9.

9. Støen Grotmol K, Gude T, Moum T, Vaglum P, Tyssen R. Risk factors at medical school for later severe depression: a 15-year longitudinal, nationwide study (NORDOC). J Affect Disord [Internet]. Elsevier. 2013. 146(1) [cited 2014 Jul 28];106-11. Available from: http://www.ncbi.nlm.nih. gov/pubmed/23017539.

10. Hawton K, Simkin S. Suicide in doctors A psychological autopsy study. 2004;57:1-4.

11. Kamski L, Frank E, Wenzel V. Suicide in medical students: case series. Anaesthesist [Internet]. 2012.61(11) [cited 2014 Jul 28];984-8. Available from: http://www.ncbi.nlm.nih. gov/pubmed/23135770

12. Martinac M, Sakić M, Skobić HJM. Suicidal ideation and medical profession: from medical students to hospital physicians. Psychiatr Danub. 2003;15(3-4):185-8.

13. Goebert D, Thompson D, Takeshita J, Beach C, Bryson P, Ephgrave K, et al. Depressive Symptoms in Medical Students and Residents : A Multischool Study. 2009;84(2):23641.

14. Viviane Franco da Silva VF, Oliveira HB, Botega NJ, Marín-León L, Barros MBA, Dalgalarrondo P. Fatores associados à ideação suicida na comunidade: um estudo de caso-controle. Cad Saúde Pública 2006; 22(9):1835-43.

15. Tyssen R, Vaglum P, Grønvold NT, Ekeberg Ø. Suicidal ideation among medical students and young physicians: a nationwide and prospective study of prevalence and predictors. 2001;64:69-79.

16. Reimer C1, Trinkaus S JH. Suicidal tendencies of physicians - an overview. Psychiatr Prax. 2005;32(8):381-5.

17. Rezende CHA, Abrão CB, Coelho EP, Passos LBS. Prevalência de sintomas depressivos entre estudantes de Medicina da Universidade Federal de Uberlândia. Revista Brasileira de Educação Médica.2008; 32(3):315-323.
18. Alexandrino-Silva C, Lazarini M, Pereira G, Bustamante C, Corrêa A, Ferraz DT, et al. Suicidal ideation among students enrolled in healthcare training programs : a cross-sectional study Ideação suicida entre estudantes da área da saúde : um estudo transversal. 2009;31(4):338-44.

19. Baldassin S, Andrade AG De. Anxiety traits among medical students. 2006;31(1):27-31.

20. Tyssen R, Hem E, Vaglum P, Grønvold NT, Ekeberg Ø. The process of suicidal planning among medical doctors: predictors in a longitudinal Norwegian sample. J Affect Disord [Internet]. 2004.80(2-3) [cited 2014 Jul 28];191-8. Available from: http://www.ncbi.nlm.nih.gov/pubmed/15207932

21. Meleiro AMAS. Suicídio entre médicos e estudantes de medicina. Rev Assoc Med Bras [Internet]. 1998;44(2):13540. Available from: http://www.scielo.br/scielo. php?script=sci_arttext\&pid=S0104-42301998000200012\&ln $\mathrm{g}=\mathrm{pt \& nrm}=$ iso\&tlng=pt.

22. Püschel KSS. Not enough help for themselves--the risk of physicians to commit suicide. Arch Kriminol. 2006;218(34):89-99.

23. Psicologia CF. O Suicídio e os Desafios para a Psicologia. Brasília; 2013.

24. Associação Brasileira de Psiquiatria. Comissão de Estudos e Prevenção do Suicídio. Suicídio: informando para prevenir. Brasília: CBM/ABP; 2014.

25. Siqueira-Batista R, Schramm FR. Eutanásia: pelas veredas da morte e da autonomia. Ciência \& Saúde Coletiva. 2004;9(1):31-41.

26. Medicina ADE. A ssistência psicológica ao estud ante de medicina : 21 anos de experiência estudante. 2008;54(1):90-4.

\section{CONTRIBUIÇÃO DOS AUTORES}

Todos os autores do manuscrito contribuíram significativamente na concepção e no desenho do estudo. Ambos contribuíram na análise e interpretação dos dados. Nathália Della Santa executou a busca nos bancos de dados e elaborou o texto. Ambos os autores revisaram criticamente o seu conteúdo intelectual e aprovaram sua versão final para ser publicada.

\section{CONFLITO DE INTERESSES}

Todos os autores do manuscrito afirmam que não têm qualquer conflito de interesse com o tema abordado no artigo, nem com os produtos/itens citados. Declaram que este artigo é original e que o trabalho, em parte ou na íntegra, ou qualquer outro trabalho com conteúdo substancialmente similar, não será enviado a outro periódico científico enquanto sua publicação estiver sendo considerada pela Revista Brasileira de Educação Médica. 


\section{ENDEREÇO PARA CORRESPONDÊNCIA}

Nathália Della Santa

Universidade Federal de Pernambuco

Programa de Pós-Graduação de Neuropsiquiatria e Ciências

do Comportamento

Av. Prof. Moraes Rêgo, 1235

Cidade Universitária - Recife

CEP 50670-901 PE

E-mail: nath.dellasanta@gmail.com 